\title{
Evaluación del plan curricular de un programa de posgrado en Ciencias de la Salud
}

José Amado* 1,2,3,a; Natalia Rodriguez 2,b; Teodoro Oscanoa 1,4,a

RESUMEN

Objetivo: Evaluar la validez interna del currículo de un programa de posgrado en ciencias de la salud.

Materiales y métodos: Estudio descriptivo con análisis documental de un programa de maestría en Docencia e Investigación en Salud de una universidad pública. Se analizó la validez interna mediante identificación de indicadores del perfil profesional, se calificaron los sílabos de las asignaturas y se valoró la congruencia en mapa curricular. Se consideraron indicadores de personalidad (alfa) y ocupacionales (beta). Se calculó estadística descriptiva con Microsoft Excel 2013. Resultados: Se identificaron indicadores alfa (diseña, demuestra, valora y aplica) e indicadores beta (líder, investigador, docente y evaluador). Los sílabos tuvieron calificación aprobatoria, pero deficiencias en metodología, materiales y bibliografía. Los indicadores conocimiento, aplicación, ser investigador y docente, estaban articulados en el mapa curricular, y se detectó desarticulación de indicadores de valoración, comunicación, ser líder y evaluador.

Conclusiones: El perfil profesional planteado está representado en el plan curricular, pero los indicadores no están articulados adecuadamente.

Palabras clave: Educación de posgrado; Ciencias de la salud; Curriculum; Evaluación educacional (Fuente: DeCS BIREME).

\section{Evaluation of a health sciences graduate program curriculum}

\section{ABSTRACT}

Objective: To evaluate the internal validity of a health sciences graduate program curriculum.

Materials and methods: A descriptive study using a documentary analysis of a public university's Master of Education and Health Research Program. The internal validity was analyzed through the identification of professional profile indicators, subjects' syllabi were rated, and the congruence in the curriculum map was assessed. Personality (alpha) and occupational (beta) indicators were considered. Descriptive statistics was performed using Microsoft Excel 2013.

Results: Alpha indicators such as the ability to design, demonstrate, assess and apply, and beta indicators such as being a leader, researcher, professor and assessor were identified. The syllabi achieved a passing grade, but deficiencies in the methodology, materials and bibliography were observed. The indicators "knowledge", "application", "being a researcher and professor" were interrelated in the curriculum map; however, indicators such as "assessment", "communication", "being a leader and assessor" were not interrelated.

Conclusions: The proposed professional profile is represented in the curriculum, but the indicators are not adequately interrelated.

Keywords: Education, graduate; Health sciences; Curriculum; Educational measurement (Source: MeSH NLM).

1. Universidad de San Martín de Porres, Facultad de Medicina Humana, Instituto de Investigación. Lima, Perú.

2. Universidad Nacional Mayor de San Marcos, Facultad de Medicina, Escuela de Posgrado. Lima, Perú.

3. Hospital Hospital Nacional Edgardo Rebagliati Martins, EsSalud. Lima, Perú.

4. Hospital Guillermo Almenara Irigoyen, EsSalud. Lima, Perú.

a. Doctor en Medicina, Médico Internista.

b. Doctora en Educación.

*Autor corresponsal. 


\section{INTRODUCCIÓN}

La educación busca elevar la condición humana en forma integral para contribuir al desarrollo y sostenibilidad del país desde los puntos de vista humanístico, científico, económico, histórico y social. La educación universitaria es la máxima jerarquía que produce conocimiento, investiga, innova y forma profesionales de alto nivel ${ }^{(1-4)}$.

El proceso de Enseñanza-Aprendizaje, desarrollado en niños y adolescentes de niveles primario o secundario (pedagogía), cambia en la universidad, especialmente en posgrado, a un proceso de Orientación-Aprendizaje esencialmente andragógico, donde tanto educador (facilitador) como educando (participante) son personas adultas con valiosa experiencia vivencial, lo que produce una interacción didáctica horizontal ${ }^{(5)}$.

La evaluación en educación médica y ciencias de la salud es un campo muy amplio, complejo y cambiante, que interviene desde el aprendizaje individual hasta los sistemas y políticas educativas ${ }^{(2,3)}$. El currículo define y estructura un programa educativo basado en objetivos educativos o competencias, compuestos por conocimientos, habilidades y actitudes, que no son evaluables o medibles como simple suma de sus componentes ${ }^{(4)}$.

El currículo con validez total (para alcanzar la excelencia) debe incluir validez interna y validez externa. La validez externa está dada por la congruencia, factibilidad, continuidad e integración del currículo con la realidad, relacionada con la cultura, demanda social, competencias exigidas y la problemática local, nacional y mundial ${ }^{(5,6)}$. La validez interna se refiere a la formulación, estructuración y relación apropiada de los elementos que integran el Plan de Estudios y las Unidades Curriculares, es decir, la inclusión de asignaturas, en relación con las exigencias del perfil profesional elegido ${ }^{(5,6)}$.

La evaluación curricular es una compleja actividad técnica y de investigación instaurada por Tyler (1949) y Taba (1962), con varios puntos de debate, cuya finalidad es conocer el funcionamiento de la situación educativa, interpretarla y plantear mejoras con adecuada retroalimentación ${ }^{(7)}$.

Desde fines del siglo $X X$, en medicina y ciencias de la salud se está implementando la educación basada en competencias, ante la necesidad de un modelo que permita articular la formación a demandas sociales y profesionales. El mercado laboral exige cada vez mayor especialización y capacitación, sobre todo en posgrado ${ }^{(8)}$.

Sin embargo, con el desarrollo industrial, la globalización y la competitividad, surge otra corriente evaluadora, la acreditación de los programas educativos, que busca calificativos, está vinculada a incentivos económicos e involucra autoridades e instituciones, pero se aleja del carácter formativo de la evaluación ${ }^{(1,7)}$.

En el Perú existen 142 universidades, públicas y privadas. El rápido crecimiento de la oferta privada ha implementado nuevos modelos que hacennecesarios mecanismos de control de calidad y reforma del sistema. La creación del Sistema Nacional de Evaluación, Acreditación y Certificación de la Calidad Educativa (SINEACE) en 2006, buscó garantizar la calidad universitaria sin éxito. La nueva ley universitaria del 2014 y la creación de la Superintendencia Nacional de Educación Superior Universitaria (SUNEDU) tratan de mejorar este problema ${ }^{(1)}$.

El presente artículo busca evaluar la validez interna del currículo de un programa de posgrado universitario y determinar la congruencia entre el perfil profesional propuesto para el egresado y la estructura curricular.

\section{MATERIALES Y MÉTODOS}

\section{Diseño y población de estudio}

Estudio de diseño descriptivo mediante revisión documental del currículo. Se evaluaron los programas universitarios de posgrado. Se seleccionó el plan de estudios de la Maestría en Docencia e Investigación en Salud de una universidad pública, dirigida a bachilleres de Medicina, Enfermería, Obstetricia, Nutrición y Tecnología médica, entre otros. Esta maestría consta de cuatro semestres académicos (18 créditos cada uno), y tres asignaturas por semestre. La modalidad de estudio fue semipresencial con dos admisiones anuales de 30 vacantes cada una. El plan se publicó en mayo de 2016.

\section{Variables y mediciones}

Se evaluó la validez interna curricular en tres momentos: identificación de indicadores prioritarios en el perfil profesional, calificación de sílabos de asignaturas y valoración de la congruencia en el mapa curricular del programa.

Del perfil profesional se seleccionaron indicadores prioritarios (alfa y beta) de acuerdo al esquema metodológico propuesto por Castro Pereira. Los indicadores alfa representan la personalidad (cualidades personales) y los beta son indicadores ocupacionales (de vigencia y prospectividad). Los indicadores alfa, a su vez, se subdividen en alfa 1 que se refiere al conocimiento, lo analítico, objetivo e investigativo; alfa 2 incluye la parte de ser comunicador, que codifique el conocimiento y lo exprese; alfa 3 involucra lo consciente, crítico, racional, sensible, lógico y ético; y alfa 4 se relaciona a la parte aplicativa, decidir y ejecutar democráticamente. Los indicadores beta incluyen rasgos deseables para cumplir funciones, roles o áreas de incumbencia ${ }^{(5,6)}$.

Para calificar sílabos de asignaturas del plan de estudios se utilizó el modelo propuesto por Rodríguez ${ }^{(5,9)}$ que emplea 
una lista de cotejo en la que se incluyen ocho áreas datos administrativos, sumilla, competencias, programación de contenidos, procedimientos didácticos, equiposmateriales, criterios de evaluación y bibliografía. Se asignó un puntaje a cada área y se presenta su porcentaje en un gráfico radial. La suma de dichas áreas fue considerada como calificación del sílabo (en sistema vigesimal).

Para la elaboración del mapa curricular se empleó una matriz con las competencias de cada asignatura ordenadas cronológicamente por semestre, que relaciona los indicadores identificados inicialmente con la competencia que los contenga mediante líneas de colores ${ }^{(6)}$.

\section{Análisis estadístico}

Para el análisis de datos se aplicó estadística descriptiva en las variables cuantitativas continuas y se agruparon los datos nominales en gráficos y líneas de tiempo. Se empleó el programa Microsoft Excel 2013.

\section{Consideraciones éticas \\ Se siguieron principios de buenas prácticas de investigación.}

\section{RESULTADOS}

Las competencias del perfil profesional del egresado de la maestría evaluada se presentan en la tabla 1.

Tabla 1. Competencias del perfil profesional del egresado de la Maestría en Docencia e Investigación en Salud

\section{Competencias del perfil profesional del egresado}

Gestiona unidades productoras de servicios educativos en salud y aplica criterios de eficiencia, calidad y equidad.

Diseña, conduce y evalúa planes macro y micro curriculares para la formación, capacitación y perfeccionamiento del potencial humano en salud.

Aplica herramientas didácticas participativas acordes con el modelo pedagógico centrado en el estudiante, basado en problemas y en evidencias, en el marco del enfoque pedagógico constructivista.

Conduce una unidad de investigación en salud y aplica la gerencia estratégica.

Diseña, ejecuta y publica investigaciones científicas en áreas relevantes de la maestría y valora el consentimiento informado.

Construye líneas de investigación en salud y evalúa investigaciones, determina su relevancia y contribución al mejoramiento de la realidad socio sanitaria del país.

Favorece el desarrollo del potencial humano en salud; tiene como ejes el liderazgo, trabajo en equipo, la educación permanente, el uso de recursos informáticos y de comunicación y la aplicación de principios bioéticos.

Fomenta y mantiene óptimas relaciones interpersonales con los miembros del equipo y la comunidad.

Demuestra capacidad creativa y de autoformación, de acuerdo a las prioridades nacionales, institucionales y profesionales.

Fuente: Página web de la maestría en Docencia e Investigación en Salud.

Los indicadores priorizados según la metodología planteada se presentan en la figura 1.

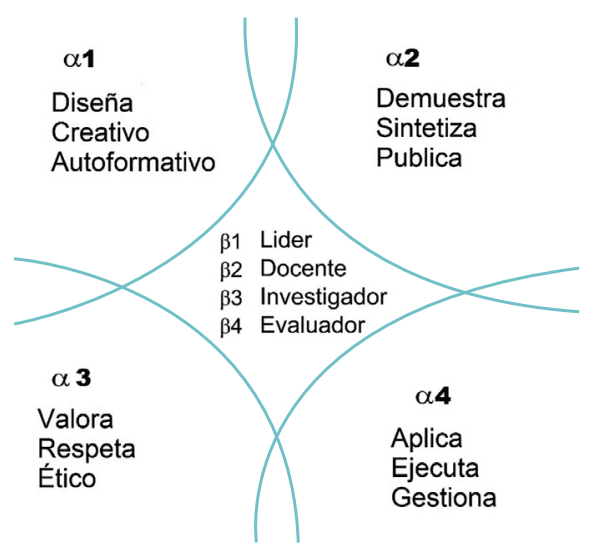

Figura 1. Indicadores alfa y beta de evaluación curricular 
La calificación promedio de los sílabos de las asignaturas fue 14,79 (rango 12,5 a 16) en el sistema vigesimal. Se encontraron dos sílabos con calificación menor a 14, que correspondieron a asignaturas del primer semestre. La máxima calificación se encontró en las asignaturas Investigación III e Investigación IV (del tercer y cuarto semestre, respectivamente). Se encontró que, para la redacción del sílabo, en la mayoría de casos, se siguió un formato prediseñado; no se especificaron adecuadamente los equipos-materiales ni la metodología, y, en varios casos, la bibliografía tuvo antigüedad mayor a 10 años y no era accesible. La calificación promedio global según áreas del sílabo se observa en la figura 2.

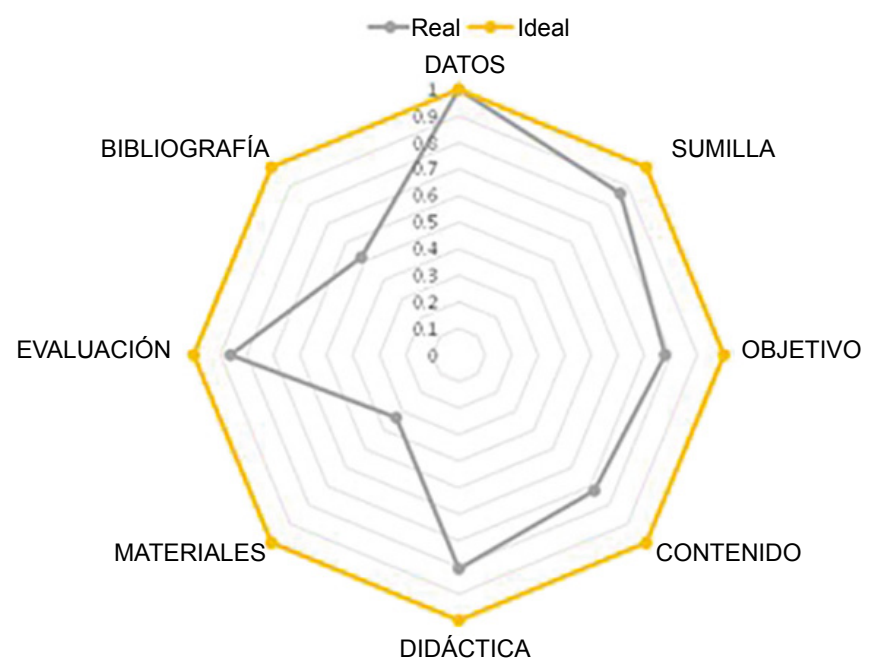

Figura 2. Resultados de calificación de sílabos

Al analizar el mapa curricular se encontró que el indicador priorizado alfa 1 está representado en las competencias planteadas de cada asignatura de todos los semestres (Figura 3). Los indicadores alfa 3 y alfa 4 están presentes en cada semestre, pero no en todas las asignaturas y el indicador alfa 2 solo está representado en las asignaturas del segundo y cuarto semestre.

\section{Semestre I}

Semestre II

Semestre III

Semestre IV

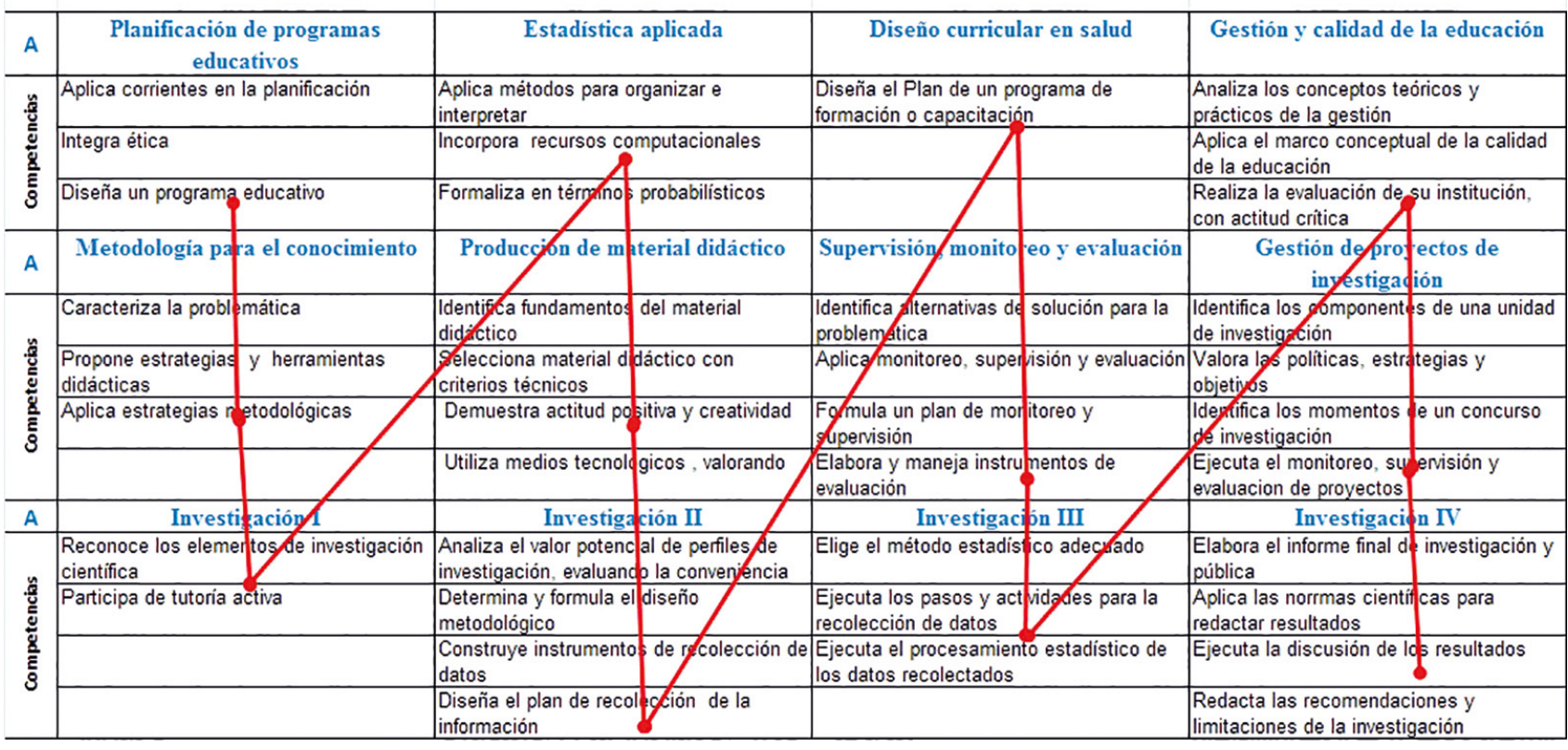

Figura 3. Indicadores alfa1 en el plan curricular del programa evaluado 
En lo referente a indicadores beta, beta 2 y beta 3 están representados en una asignatura de cada semestre de forma aislada (Figura 4), el indicador beta 4 se considera en una asignatura del tercer semestre y dos del cuarto, y el indicador beta 1 solo fue considerado en una asignatura del cuarto semestre.

\begin{tabular}{|c|c|c|c|c|c|}
\hline & Semestre I & Semestre II & Semestre III & Semestre IV & \\
\hline A & $\begin{array}{c}\text { Planificación de programas } \\
\text { educativos }\end{array}$ & Estadística aplicada & Diseño curricular en salud & Gestión y calidad de la educación & \\
\hline \multirow{3}{*}{ 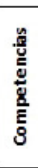 } & Aplica corrienteşen la planificación & $\begin{array}{l}\text { Aplica métodos para organizar e } \\
\text { interpretar }\end{array}$ & $\begin{array}{l}\text { Diseña el Plan de un programa de } \\
\text { formación o capacitación }\end{array}$ & $\begin{array}{l}\text { Analiza los conceptos teóricos y } \\
\text { prácticos de la gestión }\end{array}$ & \multirow{3}{*}{ B2 } \\
\hline & Integra ética & Incorpora recursos computacionales & & $\begin{array}{l}\text { Aplica el marge concentual de la calidad } \\
\text { de la educación }\end{array}$ & \\
\hline & Diseña un programa educativo & Formaliza en términos probabilísticos & & $\begin{array}{l}\text { Realizgla evaluación de su institución, } \\
\text { cop actitud crítica }\end{array}$ & \\
\hline A & Metodología para el conocimiento & Producción de material didáctico & Supervisión, monitoreo y evaluación & $\begin{array}{c}\text { Gestión de proyectos de } \\
\text { investigación }\end{array}$ & \\
\hline \multirow{4}{*}{ 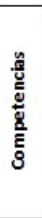 } & Caracteriza la prpblemática & $\begin{array}{l}\text { Identifica fundamentos del material } \\
\text { didáctico }\end{array}$ & $\begin{array}{l}\text { Identifica alternativas de solución para la } \\
\text { problemática }\end{array}$ & $\begin{array}{l}\text { Identifica los componentes de una unidad } \\
\text { de investigación }\end{array}$ & \\
\hline & $\begin{array}{l}\text { Propone estrate jas y herramientas } \\
\text { didácticas }\end{array}$ & $\begin{array}{l}\text { Selecciona material didáctico con } \\
\text { criterios técnicos }\end{array}$ & Aplica monitoreo, supervigion y evaluación & $\begin{array}{l}\text { Valora las políticas, estrategias y } \\
\text { objetivos }\end{array}$ & \\
\hline & Aplica estrategias metodológicas & Demuestra actitud positiva y creatividad & $\begin{array}{l}\text { Formula un_plan de monitoreo y } \\
\text { supervisión }\end{array}$ & $\begin{array}{l}\text { Identifica los momentos de un concurso } \\
\text { de investigación }\end{array}$ & \\
\hline & & Utiliza medios tecnológicos, valorando & $\begin{array}{l}\text { Elabora y maneja instrumentos de } \\
\text { evaluación }\end{array}$ & $\begin{array}{l}\text { Ejecuta el monitoreo, supervisión y } \\
\text { evaluacion de proyectos }\end{array}$ & \\
\hline A & Investigación I & Investigación II & Investigación III & Investigación IV & \\
\hline \multirow{4}{*}{ 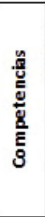 } & $\begin{array}{l}\text { Reconoce los elementos de investigación } \\
\text { cientıilca }\end{array}$ & $\begin{array}{l}\text { Analiza el valor potencial de perfiles de } \\
\text { investigacion, evaluando la conveniencia } \\
\end{array}$ & Elige el método estadístico adecuado & $\begin{array}{l}\text { Elabora el informe final de investigación y } \\
\text { pública }\end{array}$ & \\
\hline & Participa de tutoría activa & $\begin{array}{l}\text { Determina y formula el diseño } \\
\text { metodológico }\end{array}$ & $\begin{array}{l}\text { Cjecuta lns pasos y actividades para la } \\
\text { recolección de datos }\end{array}$ & $\begin{array}{l}\text { Aplica las norrmas científicas para } \\
\text { redactar resultados }\end{array}$ & E \\
\hline & & $\begin{array}{l}\text { Construye instrumentos de recolección de } \\
\text { datos }\end{array}$ & $\begin{array}{l}\text { Ejecuta el procesamiento estadístico de } \\
\text { los datos recolectados }\end{array}$ & Ejecuta la uisoución de ios resultados & \\
\hline & & $\begin{array}{l}\text { Diseña el plan de recolección de la } \\
\text { información }\end{array}$ & & $\begin{array}{l}\text { Redacta las recomendaciones y } \\
\text { limitaciones de la investigación }\end{array}$ & \\
\hline
\end{tabular}

Figura 4. Indicadores beta2 y beta3 en el plan curricular del programa evaluado

\section{DISCUSIÓN}

La demanda de capacitación ha generado un crecimiento vertiginoso de la oferta de estudios de posgrado en nuestro medio. En Perú, similar a lo reportado en Colombia, al inicio tuvo mayor desarrollo la especialización profesional (especialidades o subespecialidades) que la académica (maestrías y doctorados), con diferencias en calidad y exigencias ${ }^{(10)}$. Actualmente, se hace imprescindible tener una maestría, sobre todo en puestos universitarios. La falta de evaluación y control ha generado programas con serias deficiencias, que se pretenden erradicar con la reforma universitaria emprendida en Perú desde el $2004{ }^{(1)}$.

Específicamente, la maestría en docencia universitaria en salud está dirigida a un grupo diverso de profesionales (médicos, enfermeras, obstetras, nutricionistas, odontólogos o tecnólogos médicos), lo que dificulta la uniformidad de criterios, pero a la vez enriquece el trabajo multidisciplinario ${ }^{(8,11)}$. Esta situación es propia de las ciencias sociales y humanidades, pero en salud existe poca experiencia (12), aunque se han reportado buenos resultados en un programa cubano que por más de una década viene contribuyendo al desarrollo científico de la esfera educativa en salud, con egresados de varios países de Latinoamérica ${ }^{(13)}$.

El currículo, más que un listado secuencial de asignaturas, es un proceso que articula los contenidos, competencias, estrategias, metodología, evaluación y recursos para lograr un perfil profesional del egresado que respondan a las necesidades de la población, y debe ser constantemente analizado y actualizado ${ }^{(8)}$.

La validez interna es fundamental para asegurar la congruencia en programas de educación universitaria, por lo que el método propuesto ayudaría a detectar deficiencias y corregir resultados al aplicar el principio fundamental de la evaluación: retroalimentación y mejora de los participantes ${ }^{(5)}$; y no solo buscar la acreditación con enfoque de control, parámetros técnico-productivos y dirigidos a la parte económica ${ }^{(1,14)}$.

Al comparar esta metodología con otras propuestas para evaluar competencias, observamos que la pirámide de Miller incluye cuatro niveles (saber, saber cómo, demostrar y hacer), pero no la valoración crítica o ética, ni la forma de comunicar estos conocimientos ${ }^{(2)}$. Otro modelo de 
evaluación, el de Kirkpatrik, considera cuatro niveles, de los cuales, el 2 y 3 (evaluación del aprendizaje y la conducta) se corresponden con los de Miller; mientras que el nivel 1 (satisfacción del usuario) correspondería a los indicadores alfa 3 del modelo aplicado en la presente investigación; y la evaluación de los resultados (nivel 4) ocurriría fuera del sistema educativo y correspondería a la validez externa del currículo ${ }^{(4)}$.

Al revisar los núcleos de competencias aprobados en los Estados Unidos de Norteamérica por ACGME (Acreditation Council for Graduate Medical Education), que es el consejo acreditador de todos los programas de residencia y subespecialidad médica; observamos que el indicador alfa 1 se corresponde con el conocimiento médico, la práctica basada en el sistema y el aprendizaje; el alfa 2 con la habilidad de comunicación interpersonal; alfa 3, profesionalismo y alfa 4 , atención y cuidado al paciente $(15,16)$.

En España, para la residencia médica, se plantea el modelo de "ser médico/profesional" con criterios similares a los planteados en Norteamérica; este modelo luego se adopta para toda la Unión Europea ${ }^{(4,17)}$. En Cuba y Chile también hay reportes que definen capacidades para la especialización médica en posgrado ${ }^{(18,19)}$. Pero no se encontró reportes que evalúen competencias de programas de Maestría o Doctorado en ciencias de la salud.

En cuanto a la calificación de los sílabos, la mayoría obtuvo una puntuación aprobatoria, pero se detectaron deficiencias, sobre todo en las asignaturas del primer semestre. Las áreas con mayores problemas fueron procedimientos didácticos (estímulos), equipos y materiales (suficiencia) y bibliografía (actualidad, suficiencia y disponibilidad).

Para explicar las deficiencias que conciernen a bibliografía podemos mencionar la falta de capacitación en docencia universitaria, la baja producción científica y las escasas publicaciones de los profesores universitarios de nuestro país. Se reporta que $43,8 \%$ de los docentes universitarios de asignaturas de investigación no presenta publicaciones en Google Scholar, y solo $16,4 \%$ de los mismos ha publicado en Medline en los últimos dos años ${ }^{(20)}$. Esta brecha se estaría acortando con las diversas acciones tomadas en universidades peruanas.

El perfil profesional que plantea la Maestría muestra, al menos, un indicador alfa y un beta de los propuestos según el método de Castro-Pereyra ${ }^{(6)}$, y que se expresan claramente en la propuesta publicada,a diferencia de varios programas, de pregrado y posgrado, donde el perfil profesional no se encuentra representado en el plan de estudios vigente ${ }^{(9,11)}$.
En lo referente a la concordancia de los indicadores alfa (cualidades personales) y las competencias planteadas en el mapa curricular, se encuentra que los indicadores de conocimiento y aplicación (alfa 1 y alfa 4) están representados en todas las asignaturas de los cuatro semestres, lo que muestra una interrelación vertical y horizontal. Los indicadores de valoración y lo ético (alfa 3) faltan en dos asignaturas del primer y tercer semestre. Pero lo que más resalta es la ausencia de competencias relacionadas a la comunicación (indicador alfa 2) en todos las asignaturas del primer y tercer semestre, esto demuestra el predominio de la adquisición de conocimientos y la escasa importancia hacia la comunicación del conocimiento y publicación científica $(6,9)$.

Respecto a los indicadores beta (requerimientos ocupacionales), tanto investigador (beta 3) como docente (beta 2), se encuentran presentes en los cuatro semestres del programa; sin embargo, se evidencia la falta de articulación entre asignaturas del semestre (interrelación vertical insuficiente). El indicador beta 4 (evaluador) se presenta en los dos últimos semestres y en pocas asignaturas. El indicador beta 1 (líder) solo se menciona en una asignatura del último semestre de la Maestría, lo que refleja también la falta de importancia que tienen estos requerimientos en el programa evaluado ${ }^{(6,9)}$. Este hallazgo es similar al de una universidad cubana, que reportó una formación deficiente en habilidades científicas formativas en el currículo de pregrado ${ }^{(21)}$.

Probablemente, las deficiencias encontradas se inicien desde la creación de programas como este, pues la capacitación en docencia y didáctica en el sector salud en el Perú no es la más adecuada y muchas veces se modifica o imita programas de otras áreas sin tener todos los elementos necesarios.

Se evaluó un solo programa de posgrado, sin embargo, es un modelo práctico que puede aplicarse a otros programas educativos, a pesar de tener escasa bibliografía respecto a este método de evaluación en ciencias de la salud. En cuanto a la lista de cotejo para calificar sílabos, no se conoce su validez ni confiabilidad y debe ser mejorada.

La evaluación curricular es un proceso necesario, complejo y todavía no concluido. Debe dejar su lugar teórico y de control para desarrollar un proceso de investigación participativa y colaborativa que mejore el proceso educativo.

La validez interna en solo una parte de la evaluación curricular, sin embargo es el inicio para mejorar la calidad de nuestros programas educativos. En este momento, se necesita vivir al ritmo de mundo globalizado, con 
actualización constante del conocimiento, revisión de los programas de capacitación y estimular la difusión científica.

En conclusión, se presenta una metodología donde el perfil profesional evaluado está representado en el plan curricular, pero existen competencias desarticuladas en algunos semestres que deben mejorarse.

\section{REFERENCIAS BIBLIOGRÁFICAS}

1. Comisión Nacional de Evaluación y Acreditación Universitaria. Modelo de calidad para la acreditación de programas de posgrado, modalidad presencial y estándares para maestrías y doctorados. Ministerio de Educación Perú. Lima; CONEAU: 2010. p. Disponible en: http://www.minedu.gob.pe/ Delnteres/xtras/download.

2. Nolla-Domenjo M. La evaluación en educación médica. Principios básicos. Educ Med 2009;12(4):223-9.

3. Patel $M$. Changes to postgraduate medical education in the 21st century. Clin Med (Northfield II). 2016;16(4):311-4.

4. Oriol Bosch A. La evaluación: el reto para la educación médica del siglo XXI y Bolonia. Educ Med. 2012;15(Supl 1):S7-9.

5. Rodriguez N. Perfil profesional: Elemento fundamental del plan curricular con calidad total. Invest Educ. 2010;14(25):183-90.

6. Castro Pereira M. Modelo de Evaluación Curricular centrado en la Investigación Educativa. Material Mimeografiado. [Tesis] Caracas: Universidad de Carabobo. Facultad de Ciencias de la Salud; 1982.

7. Stenhouse L. Investigación y desarrollo del curriculum. $5^{\circ}$ Ed. Madrid: Ediciones Morata. 2003: 299-311.

8. Risco de Domínguez G. Diseño e implementación de un currículo por competencias para la formación de médicos. Rev Perú Med Exp Salud Pública 2014;31(3):572-81.

9. Gutierrez V. Diagnóstico curricular de la facultad de Enfermería de la Universidad Nacional de San Agustín y propuesta de perfil profesional. Lima: Universidad Nacional Mayor de San Marcos. Facultad de Medicina Humana; 2014.

10. Jaramillo Salazar H. La formación de posgrado en Colombia: maestrías y doctorados. Rev CTS. 2009;13(5):131-55.

11. Paredes-Bodegas E. Análisis del Proceso de Acreditación del Residentado Médico en el Perú, 2003-2005. Rev Soc Peru Med Interna. 2007;20(2):69-82.

12. Salas Perea R. ¿El rediseño curricular sin evaluación curricular es científico? Educ Médica Super. 2016;30(2):1-6.

13. Borroto Cruz ER, Salas Perea RS, Hernández García L, Fernández Oliva B, Nolla Cao N. La formación de educadores en salud y la pertinencia de la Maestría en Educación Méd. Rev Médica Super. 2012;26(4):562-75.

14. Barrios Osuna I, Roque González R. Variables de calidad para una Guía de autoevaluación de programas de posgrado en ciencias médicas. Rev Cuba Educ Medica Super. 2010;24(3):300-6.

15. Kligler B, Koithan $M$, Maizes V, Hayes $M$, Schneider C,

Lebensohn P, et al. Competency-based evaluation tools for integrative medicine training in family medicine residency: a pilot study. BMC Med Educ. 2007;7:1-11.

16. Carraccio C, Englander R, Gilhooly J, Mink R, Hofkosh $D$, Barone MA, et al. Building a Framework of Entrustable Professional Activities, Supported by Competencies and Milestones, to Bridge the Educational Continuum. Acad Med. 2017; 92(3):324-330.

17. Carreras J. Antecedentes, objetivos y contenidos de la Guía para la evaluación de competencias en medicina. Educ Med. 2012;15(Supl 1):S51-4.

18. Triviño X, Sirhan M, Moore P, Montero L. Experiencias en la implementación de un programa de formación en docencia para profesores de medicina en una universidad de Chile. Rev Perú Med Exp Salud Pública. 2014;31(3):417-23.

19. Blanco Aspiazu MA, Rodríguez Silva H, Moreno Rodríguez MA, Díaz Hernández L, Salas Perea RS, Del Pozo Jerez H, et al. Propuesta de competencias profesionales para el perfil del egresado en la especialidad de medicina interna en Cuba. Rev Haban Cienc Méd. 2015;14(6):823-38.

20. Pereyra-Elías R, Huaccho-Rojas JJ, Taype-Rondan A, Mejia $\mathrm{CR}$, Mayta-Tristán P. Publicación y factores asociados en docentes universitarios de investigación científica de escuelas de medicina del Perú. Rev Perú Med Exp Salud Pública. 2014;31(3):424-30.

21. González Capdevila O, González Franco M, Cobas Vilches ME. Las habilidades investigativas en el currículo de Medicina. Una valoración diagnóstica necesaria. Edumecentro. 2010;2(2):66-78.

\section{Fuentes de financiamiento:}

Este artículo ha sido financiado por los autores.

Conflictos de interés:

Los autores declaran no tener ningún conflicto de interés.

\section{Correspondencia:}

José Percy Amado Tineo

Dirección: Jr Belisario Florres 238 Dpto 301. Lince, Lima.

Teléfono: 2657420 - 990452547

Correo electrónico: jpamadot@gmail.com

Recibido: 11 de setiembre de 2018 . Evaluado: 09 de noviembre de 2018 Aprobado: 16 de noviembre de 2018

(c) La revista. Publicado por Universidad de San Martín de Porres, Perú. (c) $\mathbf{B y}$ Licencia de Creative Commons Artículo en acceso abierto bajo términos de Licencia Creative Commons Atribución 4.0 Internacional. (http://creativecommons.org/licenses/by/4.0/)

ORCID iDs

José Percy Amado Tineo Natalia Rodriguez del Solar Teodoro Oscanoa https://orcid.org/0000-0002-3286-4650 https://orcid.org/0000-0001-9832-4027 https://orcid.org/0000-0001-9379-4767 